\title{
Editorial
}

\section{Advances on Integrodifferential Equations and Transforms}

\author{
H. M. Srivastava, ${ }^{1}$ Xiao-Jun Yang, ${ }^{2}$ Dumitru Baleanu, ${ }^{3}$ Juan J. Nieto, ${ }^{4}$ and Jordan Hristov ${ }^{5}$ \\ ${ }^{1}$ Department of Mathematics and Statistics, University of Victoria, Victoria, BC, Canada V8W 3R4 \\ ${ }^{2}$ Department of Mathematics and Mechanics, China University of Mining and Technology, Xuzhou, Jiangsu 221008, China \\ ${ }^{3}$ Department of Mathematics and Computer Sciences, Faculty of Art and Sciences, Cankaya University, Balgat, 06530 Ankara, Turkey \\ ${ }^{4}$ Departamento de Analisis Matematico, Facultad de Matematicas, Universidad de Santiago de Compostela, 15782 Santiago, Spain \\ ${ }^{5}$ Department of Chemical Engineering, University of Chemical Technology and Metallurgy, 8 Kliment Ohridsky Boulevard, \\ 1756 Sofia, Bulgaria
}

Correspondence should be addressed to H. M. Srivastava; harimsri@math.uvic.ca

Received 3 March 2015; Accepted 3 March 2015

Copyright (C) 2015 H. M. Srivastava et al. This is an open access article distributed under the Creative Commons Attribution License, which permits unrestricted use, distribution, and reproduction in any medium, provided the original work is properly cited.

It is indeed a fairly common practice for scientific research journals and scientific research periodicals to publish special issues as well as conference proceedings. Quite frequently, these special issues are devoted exclusively to specific topics and/or are dedicated respectfully to commemorate the celebrated works of renowned research scientists. This special issue is an outcome of the ongoing importance and popularity of such topics as the theory and applications of various families of differential, integral, and integrodifferential equations as well as their fractional counterparts and associated integral and other transformations. We choose here to summarize most (if not all) of the main investigations which are contained in this special issue.

To begin with, C. Bianca et al. have investigated the existence problems for a partial integrodifferential equation with thermostat and time delay. Several Krasnoselskii type hybrid fixed point theorems together with their applications involving fractional integral equations are presented in the work by H. M. Srivastava et al. N. Wan et al. have studied the stabilized discretization in spline element method for solutions of some two-dimensional Navier-Stokes problems. Algorithmic investigation for a system of integral equations has been presented by Abdujabar Rasulov, Adem Kilicman, Zainidin Eshkuvatov, and Gulnora Raimova. I. Area et al. have derived fractional derivatives and primitives of several periodic functions. Applications of a local fractional functional method in solving diffusion equations on Cantor sets are discussed by Y. Cao et al. A study of higher-order sequential fractional differential inclusions with nonlocal three-point boundary conditions is presented by B. Ahmad and S. K. Ntouyas. D. Liu et al. have considered the Gerber-Shiu expected penalty function for the risk model with dependence and a constant dividend barrier. Some generalizations of convex functions on fractal sets are given by $\mathrm{H}$. Mo and $\mathrm{X}$. Sui. H. Guo et al. have successfully applied a Jacobicollocation method for the second kind Volterra integral equations with a smooth kernel. Solutions of initial-boundary value problems for local fractional differential equation by means of local fractional Fourier series method are presented by Y. Zhang. X.-F. Niu et al., on the other hand, have studied some local fractional derivative boundary value problems for the Tricomi equation arising in fractal transonic flow. Existence of solutions for fractional $q$-integrodifference equations with nonlocal fractional $q$-integral conditions is discussed by $S$. Asawasamrit et al. Further generalizations of the celebrated Hölder's inequality and related results on fractal space are presented by G.-S. Chen et al. Q. M. Ul Hassan et al. introduce and study an analytical technique for finding solutions for higher-order nonlinear fractional evolution equations. Applications of some expansion techniques for solving the time-fractional modified Camassa-Holm $(\mathrm{MCH})$ equation are discussed by M. Shakeel et al. N. K. Ashirbayev et al. consider the problem of solvability of an integral equation of Volterra-Wiener-Hopf type. Exact solutions of some nonlinear wave equations by the exp-function method are derived by M. Hu et al. E. Malkawi and D. Baleanu have 
investigated some fractional Killing-Yano tensors and Killing vectors using the Caputo (or, more accurately, the LiouvilleCaputo) derivative in one- and two-dimensional curved space.

\title{
Acknowledgments
}

Finally, we thank all of the participating authors and the referees for their invaluable contributions toward the remarkable success of this special issue.

\author{
H. M. Srivastava \\ Xiao-Jun Yang \\ Dumitru Baleanu \\ Juan J. Nieto \\ Jordan Hristov
}




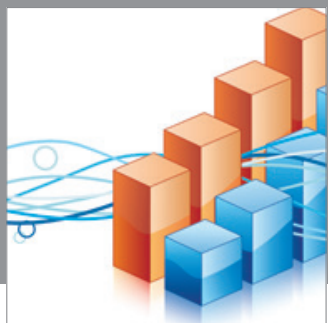

Advances in

Operations Research

mansans

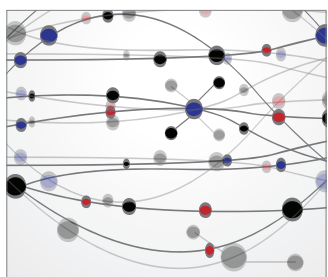

The Scientific World Journal
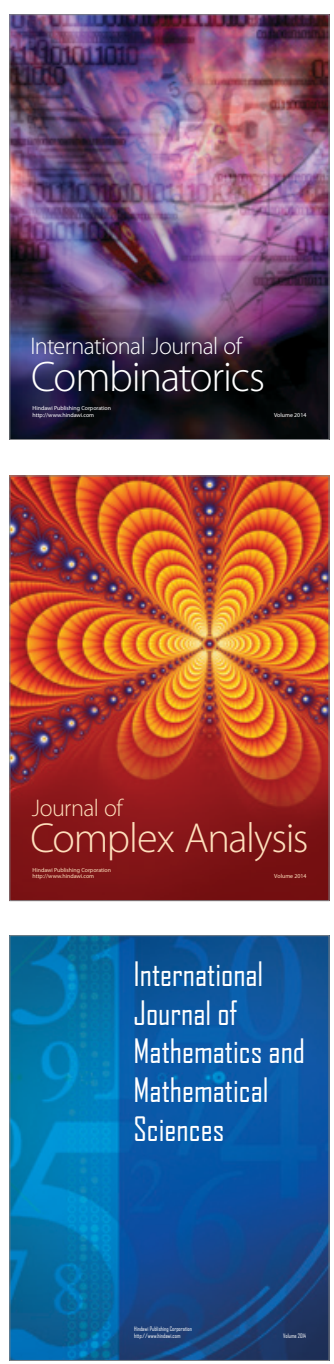
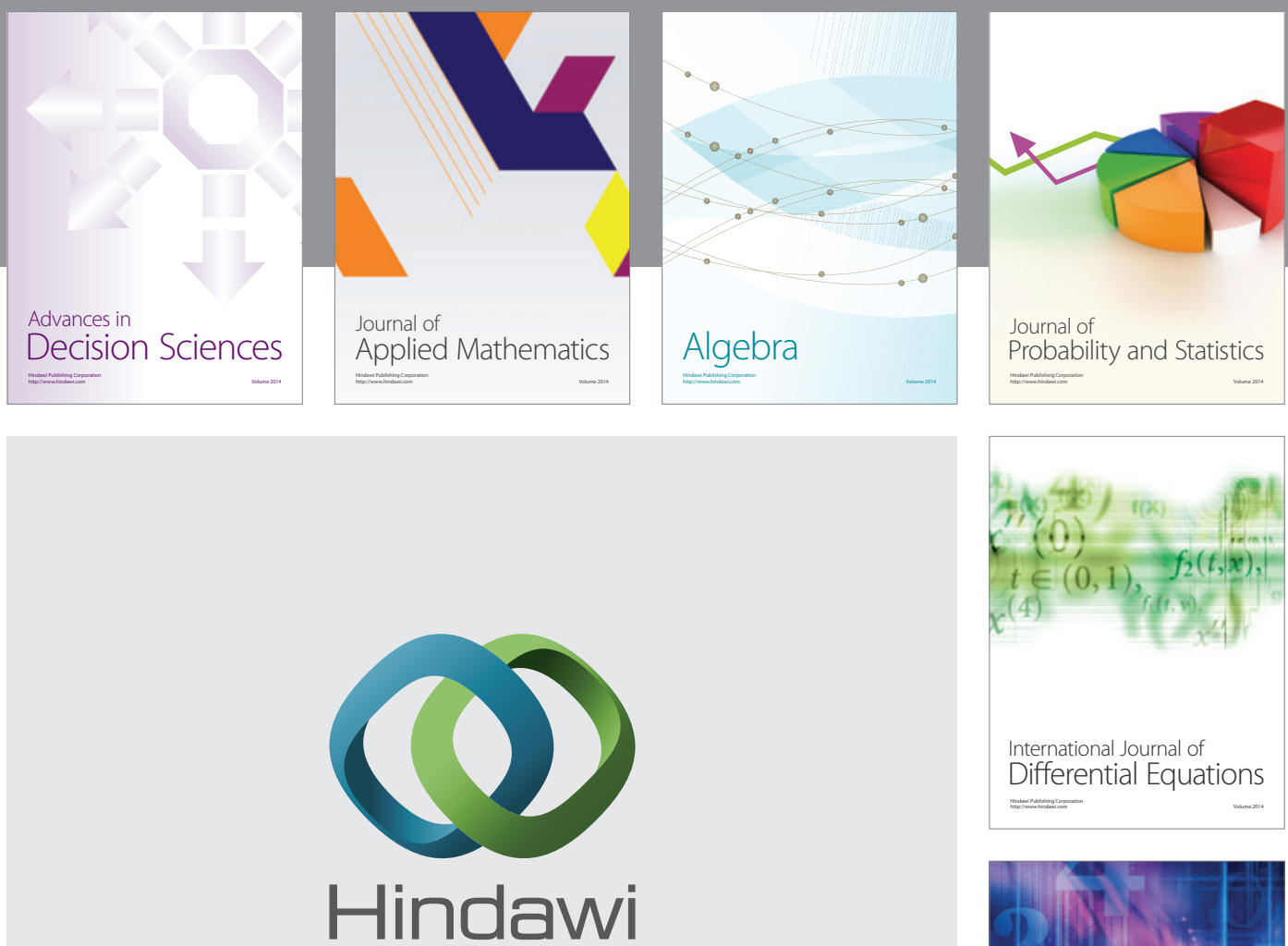

Submit your manuscripts at http://www.hindawi.com
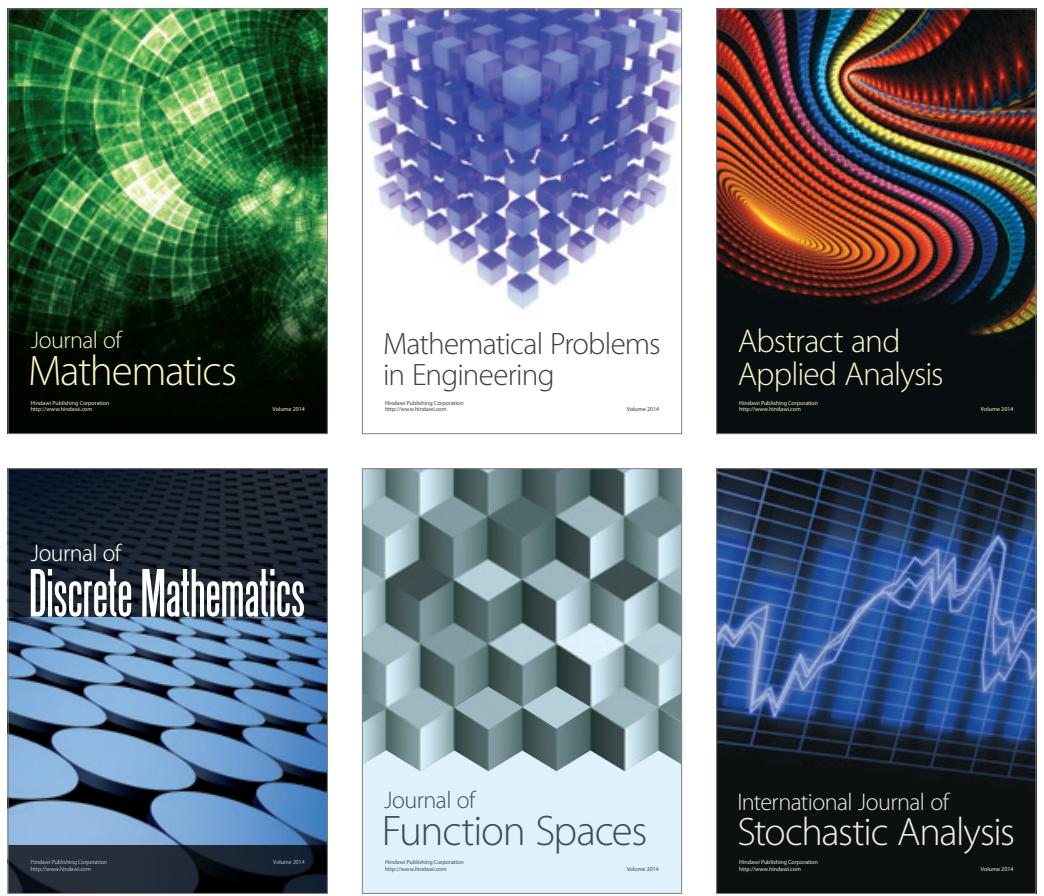

Journal of

Function Spaces

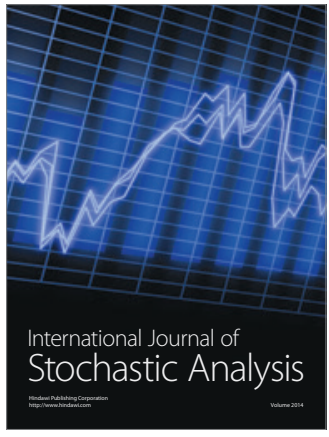

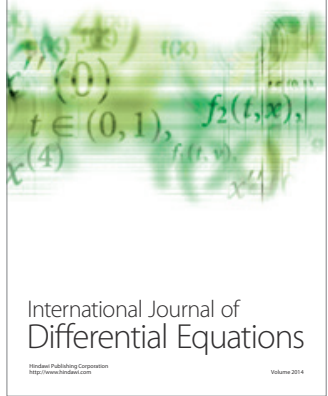
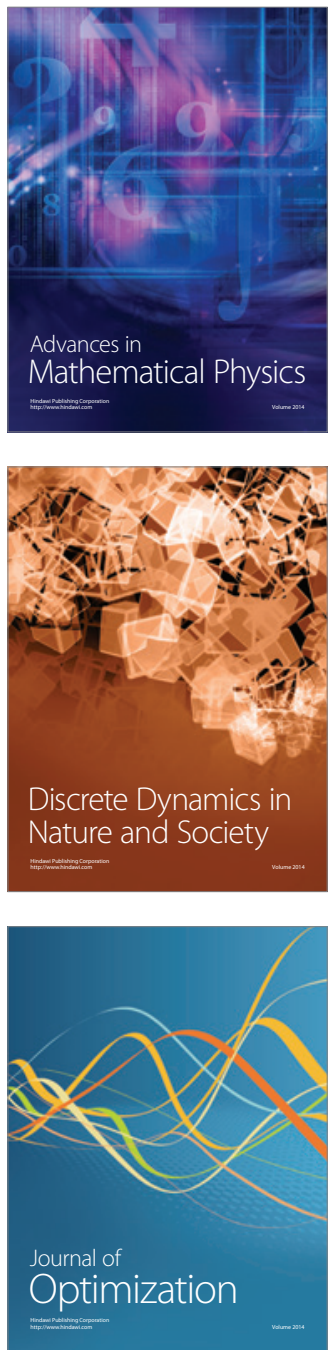